\title{
Resucitación hemostática en el paciente con choque hipovolémico hemorrágico. Reporte de un caso
}

\author{
Case report: Hemostatic resuscitation in the patient \\ with hemorrhagic hypovolemic shock
}

Julio Pablo Yáñez ${ }^{1,2}$, Miguel Calva³, Silvia Zepeda³, Rocío Garrido ${ }^{4, a}$, Fabián Fragoso Avilésª,b

\begin{abstract}
In the world, traumatic pathology continues to be a problem of great magnitude, from the point of view of public health. Today, the volumemic resuscitation in hypovolemic hemorrhagic shock is still controversial; a new alternative in volemic resuscitation is the hemostatic resuscitation that consists of the rapid use of whole blood or of the administration of the concentrate of erythrocytes (CE), fresh frozen plasma (PFC) and platelet concentrate (CP), with a fixed ratio between the products. Objective: Clinical case presentation, as well as review of the published literature on hemostatic resuscitation in patients with hemorrhagic hypovolemic shock. Material and Methods: We present the case of a female patient of 16 years of age with penetrating injuries in the neck, thorax and abdomen; management and evolution in the operating room, intensive care unit until discharge. Results: The adequate initial resuscitation in the patient with hemorrhagic hypovolemic shock has been shown to improve their survival, so that nowadays the application of new alternatives in resuscitation; As is the hemostatic resuscitation, they have yielded better results in the patient's prognosis. Conclusions: Trauma remains one of the main causes of admission to hospital units, with the young population being the most vulnerable due to car accidents. Strategies in improving the time of transfer from the accident
\end{abstract}

\section{Key words:}

Hypovolemic shock, trauma, resuscitation

\footnotetext{
Hospital Universitario de Puebla, México.

2 Servicio de Anestesiología del Hospital de Traumatología y Ortopedia "Dr. y Gral. Rafael Moreno Valle "SSA" Puebla, México

3 Benemérita Universidad Autónoma de Puebla, México.

4 Hospital General Zona Norte SSA Puebla, México.

a Licenciada en Enfermería con especialidad en Enfermería quirúrgica.

b Subdirector Médico.
}

Fecha de ingreso: 15 de agosto de 2018

Fecha de aceptación: 10 de diciembre de 2018

\section{ORCID}

https://orcid.org/0000-0003-3135-0487

Correspondencia:

Julio Cesar Pablo Yáñez

E-mail: haller_jc@hotmail.com 
site to the hospital unit, its definitive management in the operating room (if required) and the use of new alternatives in the management of hemorrhagic hypovolemic shock; such as hemostatic resuscitation, and the administration of pro-hemostatic drugs, are of great importance in the evolution and prognosis of the patient.

\section{RESUMEN}

En el mundo, la patología traumática continúa siendo un problema de gran magnitud, desde el punto de vista de salud pública. Hoy en día la resucitación volémica en el choque hemorrágico hipovolémico todavía es controversial; una nueva alternativa en la resucitación volemica es la resucitación hemostática que consiste en el uso rápido de sangre total o de la administración del concentrado de Eritrocitos (CE), Plasma Fresco Congelado (PFC) y Concentrado de Plaquetas (CP), con una razón fija entre los productos. Objetivo: Presentación de caso clínico, así como revisión de la literatura publicada sobre resucitación hemostática en el paciente con choque hipovolémico hemorrágico. Material y Métodos: Se presenta el caso de paciente femenino de 16 años de edad con lesiones penetrantes en cuello, tórax y abdomen; manejo y evolución en sala de operaciones, unidad de cuidados intensivos hasta su egreso. Resultados: La adecuada resucitación inicial en el paciente con choque hipovolémico hemorrágico ha demostrado mejorar su sobrevida, por lo que hoy en día la aplicación de nuevas alternativas en la reanimación; como es la resucitación hemostática, han arrojado mejores resultados en el pronóstico del paciente. Conclusiones: El trauma sigue siendo una de las principales causas de ingreso a las unidades hospitalarias, siendo la población joven la más vulnerable por accidentes automovilísticos. Las estrategias en la mejora del tiempo de traslado del lugar del accidente hasta la unidad hospitalaria, su manejo definitivo en sala de quirófano (si así lo requiere) y el uso de nuevas alternativas en manejo del choque hipovolémico hemorrágico; como es la resucitación hemostática, y la ministración de fármacos pro hemostáticos, resultan de gran importancia en la evolución y pronóstico del paciente.

\section{Palabras clave:}

Choque hipovolémico, trauma, resucitación

\section{Introducción}

E n el mundo, la patología traumática continúa siendo un problema de gran magnitud, desde el punto de vista de salud pública. En los últimos años se han se han descrito múltiples modificaciones en el manejo del paciente politraumatizado grave, en el cual se han desarrollado nuevos conceptos en relación con la coagulopatía inducida por el trauma (CIT), así como productos específicos relacionados con la hemostasia[1]-[6]. La resucitación volémica en el choque hemorrágico traumático todavía es controversial; tanto la conducta clásica, que es la infusión de grandes volúmenes de fluidos, como el tipo de fluido y las metas que deben alcanzar. A partir de la experiencia de la medicina en el ámbito militar se ha desarrollado el concepto de resucitación hemostática. La reanimación, en ese contexto, consiste en el uso rápido de sangre total o de la administración del concentrado de Eritrocitos (CE), Plasma Fresco Congelado (PFC) y Concentrado de Plaquetas (CP), con una razón fija y elevada entre los productos[7]-[11].

\section{Caso clínico}

Paciente femenino de 16 años de edad, que ingresa al servicio de urgencias del hospital de traumatología y ortopedia "Dr. y Gral. Rafael Moreno Valle" Puebla México; con heridas penetrantes por objeto punzo cortante. Se recibe paciente neurológicamente íntegra, al interrogatorio no refiere antecedentes de 
importancia, se realizan laboratorios iniciales (Tabla 1). A la exploración física, frecuencia cardiaca: 129 latidos por minuto, Presión arterial: 139/69 mmHg, con índice de choque de: 1,0, frecuencia respiratoria: 25 respiraciones por minuto $\mathrm{SPO}_{2}$ (saturación parcial de oxigeno): $75 \%$ con oxígeno suplementario, pupilas isocóricas, con estridor laríngeo al habla, presentando heridas penetrantes, en región central del cuello, parte anterior y posterior de hemitórax izquierdo así como en epigastrio e hipocondrio derecho, a la auscultación con murmullo vesicular izquierdo no audible, ruidos cardiacos rítmicos, peristalsis disminuida, pelvis sin alteraciones aparentes, extremidades con llenado capilar 4 segundos. Se decide manejo avanzado de vía aérea y colocación de sonda endopleural izquierda con gasto hemático de $250 \mathrm{ml}$, se canalizan dos accesos venosos periféricos y acceso venoso central subclavio izquierdo, iniciando reanimación hídrica con cristaloide (solución Hartman) 3.000 cc.

En sala de operaciones se recibe paciente bajo ventilación mecánica con ventilador de traslado, con infusión de midazolam a $25 \mathrm{cc} / \mathrm{h}$. Posteriormente se conecta a circuito de maquina de anestesia, programando ventilador con los siguientes parámetros: Modo ventilatorio (MV): Asisto Control por Volumen $(A C V)$, Volumen tidal $(\mathrm{Vt})$ : $325 \mathrm{ml}$, Frecuencia Respiratoria (FR): 12 por minuto, Fracción Inspirada de oxígeno $\left(\mathrm{FIO}_{2}\right): 60 \%$, Presión pico: $16 \mathrm{cmH}_{2} \mathrm{O}$, Dióxido de carbono espiratorio final $\left(\mathrm{ETCO}_{2}\right): 24 \mathrm{mmHg}$. En la monitorización inicial, presión arterial no invasiva (PANI): 80/42 mmHg, frecuencia cardiaca 126 latidos por minuto (lpm), índice de choque: 1,5, saturación parcial de oxígeno $\left(\mathrm{SPO}_{2}\right)$ : $98 \%$, temperatura por termómetro esofágico: $35,6{ }^{\circ} \mathrm{C}$, se monitoriza presión venosa central (PVC): $5 \mathrm{cmH}_{2} \mathrm{O}$ y se canaliza arterial radial izquierda para monitoreo hemodinámico reportando presión arterial media (PAM): $53 \mathrm{mmHg}$, variabilidad de presión de pulso (VPP): $20 \%$, variabilidad de pulso (VPS): $17 \mathrm{mmHg}$; se realiza prueba de tolerancia a volumen (positiva), con reporte posterior de PAM: $65 \mathrm{mmHg}$, VPP: $11 \%$, VPS: $9 \mathrm{mmHg}$ y PVC: $10 \mathrm{cmH}_{2} \mathrm{O}$. Se continua con infusión de midazolam a $0,5 \mathrm{mcg} / \mathrm{kg} /$ minuto, fentanil $250 \mathrm{mcg}$ y cisatracurio 6 mg endovenosos dando inicio a cirugía de reparación de daños. Durante transoperatorio se transfunden: 4 concentrados eritrocitarios (CE), 4 plasmas frescos congelados (PFC), 4 concentrados plaquetarios (CP) y 4 crioprecipitados $(\mathrm{Cr})$ en relación 1:1:1:1. Se observa mejora de los índices perfusionales, con sangrado transoperatorio de $900 \mathrm{ml}$, sin eventos adversos, se confirma por exámenes de laboratorio adecuada resucitación inicial (Tabla 2), así como la tendencia a la mejoría clínica de los signos vitales.

Al término del evento quirúrgico con TA: 110/61 PAM $77 \mathrm{mmHg} F \mathrm{~F}$ : 70 latidos por minuto (índice de choque 0,6) VPP: $8 \%$, VPS: $7 \mathrm{mmHg}$, PVC $11 \mathrm{mmHg}$. Se ingresa a la Unidad de Cuidados Intensivos, donde evolucionó favorablemente sin necesidad de vasopresor o inotrópico, con toma laboratorios de control durante su estancia (Tablas 3 y 4) siendo egresada al segundo día, posteriormente con evolución favorable, siendo egresada del hospital al onceavo día.

\section{Revisión de literatura}

Hasta hace algunos años los pacientes con trauma grave eran reanimados con gran cantidad de

\begin{tabular}{llll} 
& \multicolumn{2}{c}{ Tabla 1. Laboratorios de Ingreso a quirófano } & \\
\hline \multicolumn{1}{c}{$\begin{array}{c}\text { Citometría } \\
\text { hemática }\end{array}$} & \multicolumn{1}{c}{$\begin{array}{c}\text { Pruebas de tendencia } \\
\text { hemorrágica }\end{array}$} & \multicolumn{1}{c}{ Gasometría arterial } & \multicolumn{1}{c}{$\begin{array}{c}\text { Pruebas de } \\
\text { funcionamiento hepático }\end{array}$} \\
Leucocitos: $13,1 \times 103 / \mathrm{mm}^{3}$ & $\mathrm{TP}: 22 \mathrm{seg}$ & $\mathrm{pH} 7,33$ & $\mathrm{TGO} 65 \mathrm{IU} / \mathrm{L}$ \\
Hemoglobina: $7,5 \mathrm{~g} / \mathrm{dl}$ & $\mathrm{TPT} 55 \mathrm{seg}$ & $\mathrm{PO}_{2} 66 \mathrm{mmHg}$ & $\mathrm{TGP} 64 \mathrm{UI} / \mathrm{L}$ \\
Hematocrito: $22,2 \%$ & $\mathrm{INR} 2,3$ & $\mathrm{PCO}_{2} 55 \mathrm{mmHg}$ & $\mathrm{FA} 54 \mathrm{UI} / \mathrm{L}$ \\
Plaquetas: $80 \times 103 / \mathrm{mm}^{3}$ & Fibrinogeno:180 mg/dl & $\mathrm{HCO}_{3} 16 \mathrm{mmol} / \mathrm{L}$ & $\mathrm{BT} 2,1 \mathrm{mg} / \mathrm{dl}$ \\
& & $\mathrm{EB}-8,5 \mathrm{mmol} / \mathrm{L}$ & $\mathrm{BI} 1,7 \mathrm{mg} / \mathrm{dl}$ \\
& & $\mathrm{Lactato} 8 \mathrm{mmol} / \mathrm{L}$ & $\mathrm{BD} 0,4 \mathrm{mg} / \mathrm{dl}$ \\
& & & $\mathrm{Albúmina} 2,6 \mathrm{~g} / \mathrm{dl}$ \\
\hline
\end{tabular}

TP: Tiempo de Protrombina; TPT: Tiempo de Tromboplastina; INR: Índice Internacional Normalizado; $\mathrm{PO}_{2}$ : Presión parcial de oxígeno; $\mathrm{PCO}_{2}$ : Presión parcial de bióxido de Carbono; $\mathrm{HCO}_{3}$ : Bicarbonato; EB: Exceso de Bases; TGO: Aspartato Amino Transferasa; TGP: Alanino Amino Transferasa; FA: Fosfatasa Alcalina; BT: Bilirrubina Total; BI: Bilirrubina Indirecta; BD: Bilirrubina Directa. 


\begin{tabular}{|c|c|c|c|}
\hline Citometría hemática & $\begin{array}{c}\text { Pruebas de tendencia } \\
\text { hemorrágica }\end{array}$ & Gasometría arterial & $\begin{array}{c}\text { Pruebas de } \\
\text { funcionamiento hepático }\end{array}$ \\
\hline Leucocitos 11 mil & TP 16,1 seg & $\mathrm{pH} 7,37$ & TGO 46 IU/L \\
\hline Hemoglobina 9,5 g/dl & TPT 25 seg & $\mathrm{PO}_{2} 91 \mathrm{mmHg}$ & TGP 51 UI/L \\
\hline Hematocrito $29,2 \%$ & INR 1,6 & $\mathrm{PCO}_{2} 32 \mathrm{mmHg}$ & FA $40 \mathrm{UI} / \mathrm{L}$ \\
\hline \multirow[t]{4}{*}{ Plaquetas $110 \times 103 / \mathrm{mm}^{3}$} & Fibrinogeno 210 mg/dl & $\mathrm{HCO}_{3} 26,9 \mathrm{mmol} / \mathrm{L}$ & BT $1,8 \mathrm{mg} / \mathrm{dl}$ \\
\hline & & EB $1 \mathrm{mmol} / \mathrm{L}$ & $\mathrm{Bl} 1,4 \mathrm{mg} / \mathrm{dl}$ \\
\hline & & Lactato: $3 \mathrm{mmol} / \mathrm{L}$ & $\mathrm{BD} 0,4 \mathrm{mg} / \mathrm{dl}$ \\
\hline & & & Albumina $2,4 \mathrm{~g} / \mathrm{dl}$ \\
\hline
\end{tabular}

TP: Tiempo de Protrombina; TPT: Tiempo de Tromboplastina; INR: Índice Internacional Normalizado; PO ${ }_{2}$ : Presión parcial de oxígeno; $\mathrm{PCO}_{2}$ : Presión parcial de bióxido de Carbono; $\mathrm{HCO}_{3}$ : Bicarbonato; EB: Exceso de Bases; TGO: Aspartato Amino Transferasa; TGP: Alanino Amino Transferasa; FA: Fosfatasa Alcalina; BT: Bilirrubina Total; BI: Bilirrubina Indirecta; BD: Bilirrubina Directa.

\begin{tabular}{|c|c|c|c|}
\hline Citometria hemática & $\begin{array}{c}\text { Pruebas de tendencia } \\
\text { hemorrágica }\end{array}$ & Gasometría arterial & $\begin{array}{c}\text { Pruebas de } \\
\text { funcionamiento hepático }\end{array}$ \\
\hline Leucocitos 11,1 x103/mm3 & TP $15,1 \mathrm{seg}$ & $\mathrm{pH} 7,38$ & TGO $48 \mathrm{IU} / \mathrm{L}$ \\
\hline Hemoglobina $10,1 \mathrm{~g} / \mathrm{dl}$ & TPT 26 seg & $\mathrm{PO}_{2} 90 \mathrm{mmHg}$ & TGP $48 \mathrm{UI} / \mathrm{L}$ \\
\hline Hematocrito 31,2\% & INR 1,26 & $\mathrm{PCO}_{2} 36 \mathrm{mmHg}$ & FA $40 \mathrm{UI} / \mathrm{L}$ \\
\hline \multirow[t]{4}{*}{ Plaquetas $120 \times 103 / \mathrm{mm}^{3}$} & Fibrinogeno 610 mg/dl & $\mathrm{HCO}_{3} 25,1 \mathrm{mmol} / \mathrm{L}$ & BT $1,5 \mathrm{mg} / \mathrm{dl}$ \\
\hline & & $\mathrm{EB} 0,7 \mathrm{mmol} / \mathrm{L}$ & $\mathrm{Bl} 1,1 \mathrm{mg} / \mathrm{dl}$ \\
\hline & & Lactato: $1,5 \mathrm{mmol} / \mathrm{L}$ & $\mathrm{BD} 0,4 \mathrm{mg} / \mathrm{dl}$ \\
\hline & & & Albumina $2,6 \mathrm{~g} / \mathrm{dl}$ \\
\hline
\end{tabular}

TP: Tiempo de Protrombina; TPT: Tiempo de Tromboplastina; INR: Índice Internacional Normalizado; PO ${ }_{2}$ : Presión parcial de oxígeno; $\mathrm{PCO}_{2}$ : Presión parcial de bióxido de Carbono; $\mathrm{HCO}_{3}$ : Bicarbonato; EB: Exceso de Bases; TGO: Aspartato Amino Transferasa; TGP: Alanino Amino Transferasa; FA: Fosfatasa Alcalina; BT: Bilirrubina Total; BI: Bilirrubina Indirecta; BD: Bilirrubina Directa.

\begin{tabular}{|c|c|c|c|}
\hline Citometría hemática & $\begin{array}{c}\text { Pruebas de tendencia } \\
\text { hemorrágica }\end{array}$ & Gasometría arterial & $\begin{array}{c}\text { Pruebas de } \\
\text { funcionamiento hepático }\end{array}$ \\
\hline Leucocitos $10,5103 / \mathrm{mm}^{3}$ & TP 22,6 seg & $\mathrm{pH} \mathrm{7,45}$ & TGO $29 \mathrm{IU} / \mathrm{L}$ \\
\hline Hemoglobina 10,8 g/dl & TPT 14,8 seg & $\mathrm{PO}_{2} 96 \mathrm{mmHg}$ & TGP 54 UI/L \\
\hline Hematocrito $34,2 \%$ & INR 1,11 & $\mathrm{PCO}_{2} 38 \mathrm{mmHg}$ & FA 125 UI/L \\
\hline \multirow[t]{4}{*}{ Plaquetas $430 \times 103 / \mathrm{mm}^{3}$} & Fibrinogeno 628 mg/dl & $\mathrm{HCO}_{3} 26 \mathrm{mmol} / \mathrm{L}$ & BT $1,1 \mathrm{mg} / \mathrm{dl}$ \\
\hline & & $\mathrm{EB} 0,9 \mathrm{mmol} / \mathrm{L}$ & $\mathrm{Bl} 0,5 \mathrm{mg} / \mathrm{dl}$ \\
\hline & & Lactato: $1,3 \mathrm{mmol} / \mathrm{L}$ & $\mathrm{BD} 0,6 \mathrm{mg} / \mathrm{dl}$ \\
\hline & & & Albumina 4,3 g/dl \\
\hline
\end{tabular}

TP: Tiempo de Protrombina; TPT: Tiempo de Tromboplastina; INR: Índice Internacional Normalizado; PO $_{2}$ : Presión parcial de oxígeno; $\mathrm{PCO}_{2}$ : Presión parcial de bióxido de Carbono; $\mathrm{HCO}_{3}$ : Bicarbonato; EB: Exceso de Bases; TGO: Aspartato Amino Transferasa; TGP: Alanino Amino Transferasa; FA: Fosfatasa Alcalina; BT: Bilirrubina Total; BI: Bilirrubina Indirecta; BD: Bilirrubina Directa. 
cristaloides isotónicos y transfusión de glóbulos rojos $(G R)$, con escaso aporte de plasma, lo que generaba hemodilución y mayor coagulopatía. El uso de cristaloides en la reanimación inicial es el estándar en la práctica diaria y a la recomendación establecida por la Advanced Tauma Life Support (ATLS), así como por distintas guías de práctica clínica[12],[13],[4],[14]. No obstante, el uso de suero salino isotónico en altas dosis puede condicionar una situación de acidosis hiperclorémica. El uso de coloides no ha logrado mostrar beneficio respecto a los cristaloides en el paciente crítico. Aunque todos los fluidos son capaces de causar coagulopatia dilucional, los coloides generan problemas de la polimerización del fibrinógeno y una menor estabilidad del coagulo. Las drogas vaso activas (DVA) en el trauma grave, concretamente la noradrenalina, se utiliza en situaciones de hipotensión arterial refractaria a la resucitación con fluidos, su uso en estudios experimentales ha reflejado una reducción en los requerimientos de fluidos y menores perdidas sanguíneas, los estudios en humanos muestran resultados contradictorios e incluso su administración precoz en el trauma asocian un aumento de la mortalidad[12],[13],[4]. Dado que la corrección de los parámetros hemodinámicos de macrocirculación no garantizan la resolución de la hipoperfusión tisular, las determinaciones iniciales de lactato, déficit de bases y su aclaramiento con posterioridad, permitan identificar pacientes con resucitación incompleta o con problemas no resueltos, como lesiones graves infradiagnosticadas. Recientemente, en estudios prospectivos se ha mostrado que el aclaramiento precoz de lactato (0-2 h) es un importante factor pronóstico, por lo que podría ser de gran utilidad su seguimiento durante la resucitación del trauma[12],[13],[4],[14]. Durante los últimos años se ha desarrollado la resucitación hemostática, cuyos objetivos son minimizar el sangrado, restablecer la perfusión, prevenir la coagulopatía y evitar las disfunciones orgánicas. De esta forma, se busca disminuir la mortalidad del trauma, especialmente la muerte por sangrado no controlado[12],[13],[4],[14],[15],[16],[17],[18],[5]. Uno de los objetivos de la resucitación hemostática es prevenir o corregir el desarrollo de coagulopatía. Tradicionalmente, se consideraba que la coagulopatía del trauma era secundaria a hemodilución de factores de coagulación, acidosis e hipotermia, desarrollándose varias horas después del ingreso al hospital[19]. Debemos tener en cuenta que hasta un $25-43 \%$ de los pacientes traumatizados que llegan al hospital presentan trastornos en la coagulación[20],[21]. El origen de la CIT (Coagulopatía Inducida por el Trauma) es multifactorial, dentro de los que se pueden citar, la pérdida de factores por puntos sangrantes, la dilución de los existentes (por la infusión de fluidos o la administración de concentrados de hematíes sin asociar PFC), la activación de la fibrinólisis, la alteración de la funcionalidad de las proteasas de manera secundaria a la acidemia, la hipotermia y el shock[22].

La CIT se agrava ante la presencia de shock al ingreso hospitalario, dado a que la hipoperfusión promueve un estado de hiperfibrinolisis, de manera independiente a la presencia de hipotermia, acidosis o dilución de factores, los cuales se presentarán posteriormente en caso de una resucitación no apropiada[16],[23]. La hipotermia se ha mostrado como un factor de riesgo independiente de mortalidad en el trauma[22],[24]. La corrección de la hipotermia mejora la actividad de los tiempos de coagulación y de la actividad plaquetaria[25],[26].

El reemplazo de las pérdidas sanguíneas tras el traumatismo, mediante la transfusión de hemoderivados, ha variado en las últimas décadas. La proporción en la que estos componentes deben ser infundidos, también ha sido objeto de estudio. Recientemente se han publicado los resultados del estudio PROMMPT[20] que incluyó a 1.245 pacientes de 10 centros de trauma, mostrando una reducción de la mortalidad cuando los ratios de PFC (Plasma Fresco Congelado) y Plaquetas respecto al CE (Concentrado Eritrocitario) se aproximaba al 1:1[20]. De igual forma, el uso de crioprecipitados o fibrinógeno administrados en relación con el número de CE infundidos, también mejoran la supervivencia[27],[28]. Dentro de los fármacos prohemostáticos el estudio CRASH-2 estudió y evaluó el uso de ácido tranexámico (ATX) dentro de las primeras 8 horas del trauma, obteniendo una reducción significativa de la mortalidad global al mes y de mortalidad por hemorragia masiva respecto al placebo[29]. El uso de fibrinógeno en combinación con CE es un factor asociado a supervivencia de los pacientes[31], se aconseja su uso cuando sus niveles plasmáticos se detecten por debajo de 1,5 g/[30]. El complejo protrombínico aporta grandes dosis de factores de coagulación (II, VII, IX y X) en un volumen reducido en comparación con el PFC, indicado en pacientes que se encuentran bajo efectos de determinados fármacos anticoagulantes[31]. Por último, se ha evaluado el uso de factor VII recombinante, que ha objetivado una menor necesidad de hemoderivados, así como mejora en el sangrado en el trauma cerrado, no considerado como manejo de primera línea, por no presentar una reducción clara en la mortalidad, reservándose su uso cuando otras medidas no han sido efectivas. Dentro de los datos de laboratorio para monitorizarla coagulopatía debe tenerse en cuenta 
la determinación precoz y seriada de TTPa, tiempo de protrombina (TP), International Normalized Ratio (INR), fibrinógeno (Fb) y plaquetas. Igualmente, en pacientes quirúrgicos o traumatizados con hemorragia grave se debe valorar completar el estudio de la coagulación con determinaciones de tromboelastografía (TEG) y la tromboelastometría rotacional $\left(\right.$ ROTEM $\left.^{\circledR}\right)$, siempre teniendo en cuenta la disponibilidad en cada centro hospitalario[32].

\section{Discusión}

En el caso clínico presentado se expone a una paciente en choque hipovolémico clase IV por ATLS, traumático, el cual es manejada con transfusión transoperatoria de concentrados eritrocitarios, plasma fresco congelado, concentrado plaquetario y crioprecipitados en relación 1:1:1:1, con mejora en la estabilidad hemodinámica y perfusión tisular.

En la literatura actual y en los reportes de diversos estudios, sugieren en el manejo inicial del paciente con choque hipovolémico hemorrágico, la administración temprana de hemoderivados, así como fármacos prohemostáticos con el fin de disminuir la coagulopatía, causada por el trauma o una reanimación inadecuada mejorando así la sobrevida y el pronóstico del paciente. En nuestro caso el manejo inicial dado bajo el término de resucitación hemostática a nuestra paciente mejoró su pronóstico, así como su corta estancia en la Unidad de Cuidados Intensivos, por lo que el resultado esperado fue lo reportado en la bibliografía.

\section{Conclusiones}

Actualmente la reanimación volemica con nuevas alternativas en manejo del choque hipovolémico hemorrágico como es la resucitación hemostática, así como administración de fármacos pro hemostáticos han dado resultados favorables en la evolución y pronóstico del paciente.

\section{Referencias}

1. Jansen JO, Thomas R, Loudon MA, Brooks A. Damage control resuscitation for patients with major trauma. BMJ. 2009 Jun;338 jun05 1:b1778. https:// doi.org/10.1136/bmj.b1778 PMID:19502278

2. Holcomb JB, Jenkins $D$, Rhee $P$, Johannigman J, Mahoney P, Mehta S, et al. Damage control resuscitation: directly addressing the early coagulopathy of trauma. J Trauma. 2007 Feb;62(2):30710. https://doi.org/10.1097/ TA.0b013e3180324124 PMID:17297317

3. Shaz BH, Dente CJ, Nicholas J, MacLeod JB, Young AN, Easley $\mathrm{K}$, et al. Increased number of coagulation products in relationship to red blood cell products transfused improves mortality in trauma patients. Transfusion. 2010;50:493-500. https://doi.org/10.1111/j.15372995.2009.02414.x.
4. Spahn DR, Bouillon B, Cerny V, Coats TJ, Duranteau J, Fernández-Mondéjar E, et al. Management of bleeding and coagulopathy following major trauma: an updated European guideline. Crit Care. 2013 Apr;17(2):R76. https://doi.org/10.1186/cc12685 PMID:23601765

5. Dutton RP. Resuscitative strategies to maintain homeostasis during damage control surgery. Br J Surg. 2012 Jan;99(S1 Suppl 1):21-8. https://doi.org/10.1002/ bjs.7731 PMID:22441852

6. Beekley AC. Damage control resuscitation: a sensible approach to the exsanguinating surgical patient. Crit Care Med. 2008 Jul;36(7 Suppl):S267-74. https://doi.org/10.1097/ CCM.0b013e31817da7dc PMID:18594252

7. Nunez TC, Voskresensky IV, Dossett LA, Shinall R, Dutton WD, Cotton BA. Early prediction of massive transfusion in trauma: simple as $A B C$ (assessment of blood consumption)? J Trauma. 2009 Feb;66(2):34652. https://doi.org/10.1097/ TA.0b013e3181961c35 PMID:19204506

8. Malone DL, Hess JR, Fingerhut A. Massive transfusion practices around the globe and a suggestion for a common massive transfusion protocol. J Trauma. 2006 Jun;60(6 Suppl):S91-6. https://doi.org/10.1097/01. ta.0000199549.80731.e6 PMID:16763487

9. Miller RD, Robbins TO, Tong MJ, Barton SL. Coagulation defects associated with massive blood transfusions. Ann Surg. 1971 Nov;174(5):794-801. https:// doi.org/10.1097/00000658197111000-00010 PMID:5113461

10. perry JL, Ochoa JB, Gunn SR, Alarcon LH, Minei JP, Cuschieri J, et al.; Inflammation the Host Response to Injury Investigators. An FFP:PRBC transfusion ratio $\geq 1: 1.5$ is associated 
with a lower risk of mortality after massive transfusion. J Trauma. 2008 Nov;65(5):98693. https://doi.org/10.1097/ TA.0b013e3181878028 PMID:19001962

11. Dente CJ, Shaz BH, Nicholas JM, Harris RS, Wyrzykowski AD, Ficke $B W$, et al. Early predictors of massive transfusion in patients sustaining torso gunshot wounds in a civilian level I trauma center. J Trauma. 2010 Feb;68(2):298304. https://doi.org/10.1097/ TA.0b013e3181 cf7f2a PMID:20154541

12. Duchesne JC, McSwain NE Jr, Cotton BA, Hunt JP, Dellavolpe J, Lafaro K, et al. Damage control resuscitation: the new face of damage control. J Trauma. 2010 Oct;69(4):97690. https://doi.org/10.1097/ TA.0b013e3181f2abc9 PMID:20938283

13. Johansson Pl, Stensballe J, Ostrowski SR. Current management of massive hemorrhage in trauma. Scand J Trauma Resusc Emerg Med. 2012 Jul;20(Jul):47. https://doi.org/10.1186/17577241-20-47 PMID:22776724

14. Cotton BA, Guy JS, Morris JA Jr, Abumrad NN. The cellular, metabolic, and systemic consequences of aggressive fluid resuscitation strategies. Shock. 2006 Aug;26(2):11521. https://doi.org/10.1097/01. shk.0000209564.84822.f2 PMID:16878017

15. Kind SL, Spahn-Nett GH, Emmert MY, Eismon J, Seifert B, Spahn DR, et al. Is dilutional coagulopathy induced by different colloids reversible by replacement of fibrinogen and factor XIII concentrates? Anesth Analg. 2013 Nov;117(5):106371. https://doi.org/10.1213/ ANE.0b013e3182a52876 PMID:24029856

16. Plurad DS, Talving $P$, Lam L, Ina- ba K, Green D, Demetriades D. Early vasopressor use in critical injury is associated with mortality independent from volume status. J Trauma. 2011 Sep;71(3):56570. https://doi.org/10.1097/ TA.0b013e3182213d52 PMID:21908995

17. Régnier MA, Raux M, Le Manach Y, Asencio Y, Gaillard J, Devilliers $C$, et al. Prognostic significance of blood lactate and lactate clearance in trauma patients. Anesthesiology. 2012 Dec;117(6):1276-88. https://doi.org/10.1097/ ALN.0b013e318273349d PMID:23168430

18. Arnold TDW, Miller $M$, van Wessem KP, Evans JA, Balogh ZJ. Base Deficit From the First Peripheral Venous Sample: A Surrogate for Arterial Base Deficit in the Trauma Bay. The Journal of Trauma: Injury, Infection, and Critical Care. Ovid Technologies (Wolters Kluwer Health); 2011 Oct;71(4):793-7. Disponible en: http://dx.doi.org/10.1097/ ta.0b013e31822ad694

19. Brohi K, Singh J, Heron M, Coats T. Acute traumatic coagulopathy. J Trauma. 2003 Jun;54(6):112730. https://doi.org/10.1097/01. TA.0000069184.82147.06 PMID:12813333

20. Holcomb JB, del Junco DJ, Fox EE, Wade CE, Cohen MJ, Schreiber MA, et al. The Prospective, Observational, Multicenter, Major Trauma Transfusion (PROMMTT) Study. JAMA Surgery]. American Medical Association (AMA); 2013 Feb 1;148(2):127. Disponible en: http://dx.doi.org/10.1001/2013. jamasurg. 387

21. Maegele $M$, Lefering $R$, Yucel $N$, Tjardes $T$, Rixen $D$, Paffrath $T$, et al.; AG Polytrauma of the German Trauma Society (DGU). Early coagulopathy in multiple injury: an analy- sis from the German Trauma Registry on 8724 patients. Injury. 2007 Mar;38(3):298304. https://doi.org/10.1016/j. injury.2006.10.003 PMID:17214989

22. Hess JR, Brohi K, Dutton RP, Hauser CJ, Holcomb JB, Kluger $Y$, et al. The coagulopathy of trauma: a review of mechanisms. J Trauma. 2008 Oct;65(4):74854. https://doi.org/10.1097/ TA.0b013e3181877a9c PMID:18849786

23. Brohi K, Cohen MJ, Ganter MT, Matthay MA, Mackersie RC, Pittet J-F. Acute Traumatic Coagulopathy: Initiated by Hypoperfusion. Annals of Surgery. Ovid Technologies (Wolters Kluwer Health); 2007 May;245(5):812-8. Disponble en: http://dx.doi.org/10.1097/01. sla.0000256862.79374.31

24. Brohi $K$, Singh J, Heron M, Coats T. Acute traumatic coagulopathy. J Trauma. 2003 Jun;54(6):112730. https://doi.org/10.1097/01. TA.0000069184.82147.06 PMID:12813333

25. Holcomb JB, Jenkins $D$, Rhee $P$, Johannigman J, Mahoney P, Mehta $\mathrm{S}$, et al. Damage control resuscitation: directly addressing the early coagulopathy of trauma. J Trauma. 2007 Feb;62(2):30710. https://doi.org/10.1097/ TA.0b013e3180324124 PMID:17297317

26. Martini WZ, Pusateri AE, Uscilowicz JM, Delgado AV, Holcomb JB. Independent contributions of hypothermia and acidosis to coagulopathy in swine. J Trauma. 2005 May;58(5):10029. https://doi.org/10.1097/01. TA.0000156246.53383.9F PMID:15920416

27. B.H. Shaz,C.J. Dente,J. Nicholas,J.B. MacLeod,A.N. Young, K. Easley. Increased number of coagulation products in relationship to red blood cell 
products transfused improves mortality in trauma patients https://doi.org/10.1111/j.15372995.2009.02414.x.

28. H.K. Stinger, P.C. Spinella,J.G Perkins, K.W. Grathwohl,J. Salinas, W.Z. Martini. The ratio of fibrinogen to red cells transfused affects survival in casualties receiving massive transfusions at an army combat support hospital. https://doi.org/10.1097/ TA.0b013e318160a57b.

29. H. Shakur, I. Roberts, R. Bautista, J. Caballero, T. Coats, Y. Dewan, CRASH-2 trial collaborators. Effects of tranexamic acid on death, vascular occlusive events, and blood transfusion in trauma patients with significant haemorrhage (CRASH-2): A randomised, placebo-controlled trial. Disponible en: http:// dx.doi.org/10.1016/s01406736(10)60835-5

30. Schöchl H, Nienaber U, Hofer G, Voelckel W, Jambor C, Scharbert G, et al. Goal-directed coagulation management of major trauma patients using thromboelastometry (ROTEM $\AA$ )-guided administration of fibrinogen concentrate and prothrombin complex concentrate. Critical Care. Springer Nature; 2010;14(2):R55. Disponible en: http://dx.doi.org/10.1186/ cc8948

31. Fernández-Hinojosa E, MurilloCabezas F, Puppo-Moreno A, Leal-Noval SR. [Treatment alternatives in massive hemorrhage]. Med Intensiva. 2012 Oct;36(7):496-503. PMID:22321860

32. Boffard KD, Choong PI, Kluger $Y$, Riou B, Rizoli SB, Rossaint $R$, et al.; NovoSeven Trauma Study Group. The treatment of bleeding is to stop the bleeding! Treatment of trauma-related hemorrhage. Transfusion. 2009 Dec;49 Suppl 5:240S-7S. https://doi.org/10.1111/ j.1537-2995.2008.01987.x PMID:19954486 